\title{
Fast synchronous acquisition method using chaos DOS-CDMA in Software Definable Radio Networks
}

\author{
Hajime Yamasaki ${ }^{a}$, Takeshi Higashino, Katsutoshi Tsukamoto, \\ and Shozo Komaki
}

Division of Electrical, Electronic and Information Engineering, Graduate School of Engineering, Osaka University

2-1 Yamada-oka, Suita, Osaka, 565-0871, Japan

a)yamasaki@roms.comm.eng.osaka-u.ac.jp

Abstract: A fast synchronous acquisition method which is applied to Direct Optical Switching-Code Division Multiple Access (DOS-CDMA) scheme in Software Definable Radio Networks (SDRNs) is proposed. This paper shows that the chaotic synchronization enables to reduce synchronous acquisition time in DOS-CDMA scheme compared to the sliding correlation method.

In case that code length is 121, reduction effect of synchronous acquisition time of $95 \%$ is obtained by the computer simulations.

Keywords: Software Definable Radio Networks (SDRNs), chaos DOSCDMA, synchronous acquisition time, chaotic synchronization

Classification: Microwave and millimeter wave devices, circuits, and systems

\section{References}

[1] S. Komaki, "Microwave technologies for Software Radio Networks," Proc. APMC2003, vol. 3, pp. 1780-1785, Nov. 2003.

[2] K. Tsukamoto et al, "Direct optical switching code-division multipleaccess system for fiber-optic radio highway networks," J. Lightwave Technol., vol. 21, no. 12, pp. 3209-3220, Dec. 2003.

[3] G. Marubayashi, "Recent Research and Development Activities on Spread Spectrum Communication Systems," IEICE Trans., vol. J74-B2, no. 5, pp. 176-181, May 1991.

[4] N. Oketani and T. Ushio, "Chaotic communication method using globally coupled maps," IEICE Trans. Fundamentals, vol. J79-A, no. 8, pp. 14271432, Aug. 1996.

[5] K. Umeno et al., "Theoretical Analysis of Chaos CDMA: Chaos versus Optimal Binary PN Sequences," IEICE Gen. Conf., SAB-1-3, pp. 508509, March 1999. 


\section{Introduction}

Recently, Software Definable Radio Networks (SDRNs) have been studied in order to meet transmission for different kind of wireless service [1]. In the SDRN, Radio-on-Fiber (RoF) technique plays an important role. RoF technique can transmit wireless services with keeping a radio modulation format because of huge bandwidth and low loss characteristics. The SDRN is composed of two or more Control Stations (CS) and Radio Base Stations (RBS), and there are connected through routing nodes (RN) in order to forward wireless service to suitable CS or SDR Gateway. The Direct Optical Switching-Code Division Multiple Access (DOS-CDMA) has been studied as a multiple access scheme [2]. The DOS-CDMA scheme encodes a radio frequency (RF) signal in optical domain using an optical switch (OSW) device. Therefore, assigning a unique code sequence for every wireless service, the DOS-CDMA scheme can be used as a routing method.

Figure 1 shows the configuration of RBS and RN. At the RBS, received $\mathrm{RF}$ signals are divided by the use of band pass filter (BPF). Each RF signal is converted into an intensity modulation (IM) signal. IM signal is encoded at OSW which is driven by the electric code sequence. The CS and the SDR gateway can be identified by code sequence. Obtained IM/CDMA signals are multiplexed by the use of optical coupler.

In the RN, IM/CDMA signals are divided into two branches. One side is photodetected and then baseband Pulse Amplitude Modulation (PAM) /CDMA signal is obtained for input of synchronous acquisition circuit. Synchronous acquisition circuit matches the timing between local code sequences and received baseband PAM/CDMA signal within half of chip width. Synchronous tracking circuit maintains acquired synchronous state. A trigger signal sends to code sequence generators. Another IM-CDMA signal is delayed and then decoded by the use of OSW which is driven by code sequence. The output signal is transfered to the next node. There is sliding correlation as a conventional synchronous acquisition method [3].

In this paper, a fast synchronous acquisition method which is applied to conventional DOS-CDMA method in the SDRN is proposed. The proposed method is based on the chaotic synchronization [4]. Thus, it is called as chaos DOS-CDMA method. The correlation characteristic of the chaos code is almost the same as other code sequences [5]. In addition, since computer processing can perform synchronous acquisition process, the composition of the RN can be expected to simplify compared with the sliding correlation method using prime code.

The reduction of synchronous acquisition time is examined by the computer simulation.

\section{Chaos DOS-CDMA method}

Figure 2 (a) shows the configuration of RBS in chaos DOS-CDMA method. Unique chaotic generating systems, $S_{i}(i=1,2, \ldots, N)$, are assigned for every wireless service. $S_{i}$ is also assigned to every destination. $S_{i}$ is constituted by 


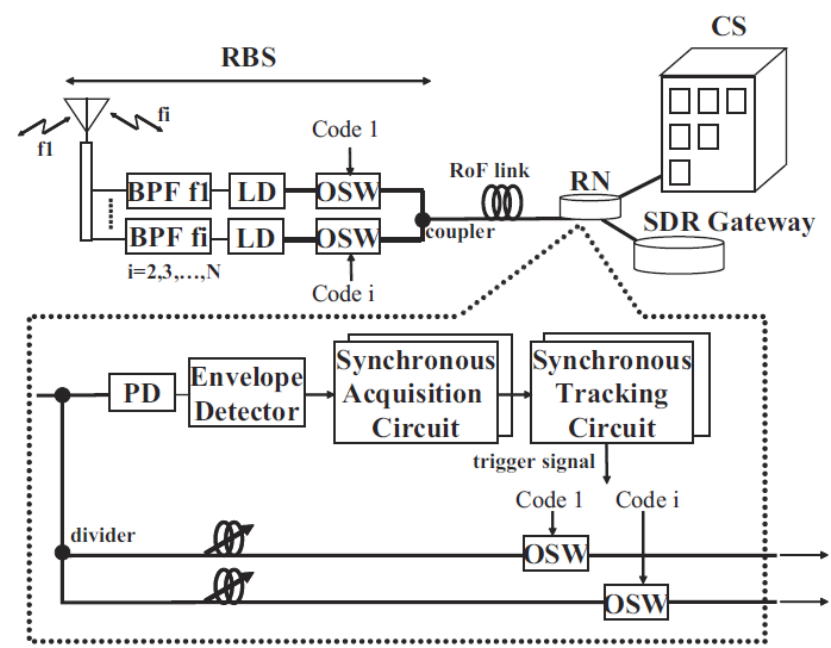

Fig. 1. Configuration of RBS and RN

a chaotic generating function, $G_{i}()$, and a parameter, $a_{i}$. The variable, $x_{i}(k)$, is computed from $G_{i}()$. In this paper, what is converted $x_{i}(k)$ into binary sequence is denoted as chaotic sequence, $\tilde{x}_{i}(k)$. The sum of $\tilde{x}_{i}(k)$ on $i$ is fed back to $S_{i}$, then $\tilde{x}_{i}(k+1)$ is calculated in $k+1$ th step. IM signal is encoded by $\tilde{x_{i}}(k)$. The $k$-th value of optical intensity is equivalent to the sum of $\tilde{x}_{i}(k)$ on $i$. The length of chaotic sequence is able to have an arbitrary integer value. However, since an RF signal is overlapped on the optical intensity, an exact recovery is difficult for a non-periodicity signal. Therefore, code length, $L$, is introduced to chaotic sequence. That is, $\tilde{x}_{i}(k)(0 \leq k \leq L-1)$ is saved, then $\tilde{x}_{i}(k)$ is repeated with its period of $T_{F}$.

Figure 2 (b) shows the configuration of RN in chaos DOS-CDMA method. In synchronous acquisition process, first, the amplitude value of the received baseband PAM/CDMA signal is detected, and inputs $S_{i}$. Chaotic synchronization [4] is achieved by calculation of chaotic sequence, $\tilde{y}_{i}(k)$, of the RN $\left(\tilde{x}_{i}(k)=\tilde{y}_{i}(k)\right)$. Correlation detection of $\tilde{y}_{i}(k)$ and the amplitude value of the received baseband PAM/CDMA signal is performed at intervals of every chip width, $T_{c}$, and a threshold value judging is performed based on the correlator output value, $v_{i}(m)\left(m\right.$ : the number of chip) at intervals of every $T_{c}$, in the period of $T_{F}$.

In the case of prime code, when the weight (the number of " 1 " in the code sequence) and $L$ is decided, error judging will not occur since the greatest value of cross correlation can be estimated. However, in the case of chaotic sequence, the weight changes according to an initial value in $S_{i}$. Therefore, it is necessary to estimate the relationship between error judging rate and possible range of a threshold value, $T h$. The error judging rate is the sum of the probability Eq. (1).

$$
P\left(\frac{\tilde{x}_{i}(k) \neq \tilde{y}_{i}(k)}{v_{i}(m)>T h}\right)+P\left(v_{i}(L)<T h\right)
$$

Also, the calculation of the average synchronous acquisition time, $T_{S A}$, differs with prime code and chaotic sequence. In the case of prime code, a threshold value judging is performed at intervals of every $T_{F}$. However, in 


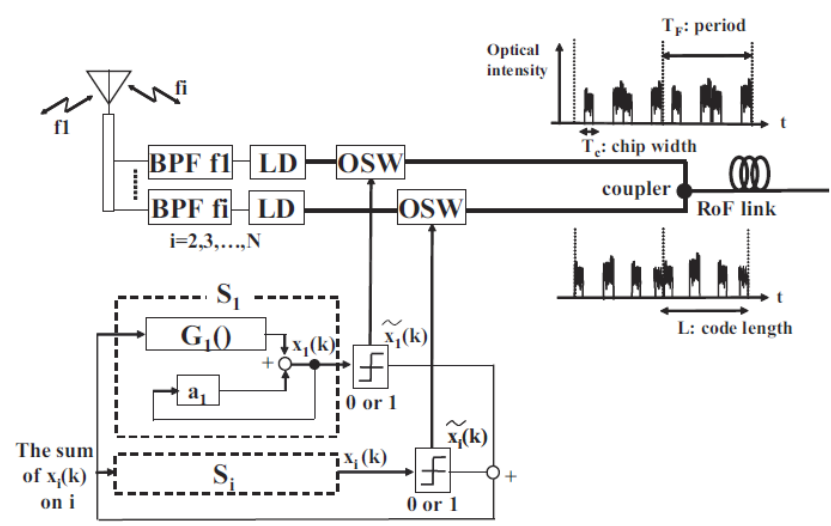

(a)

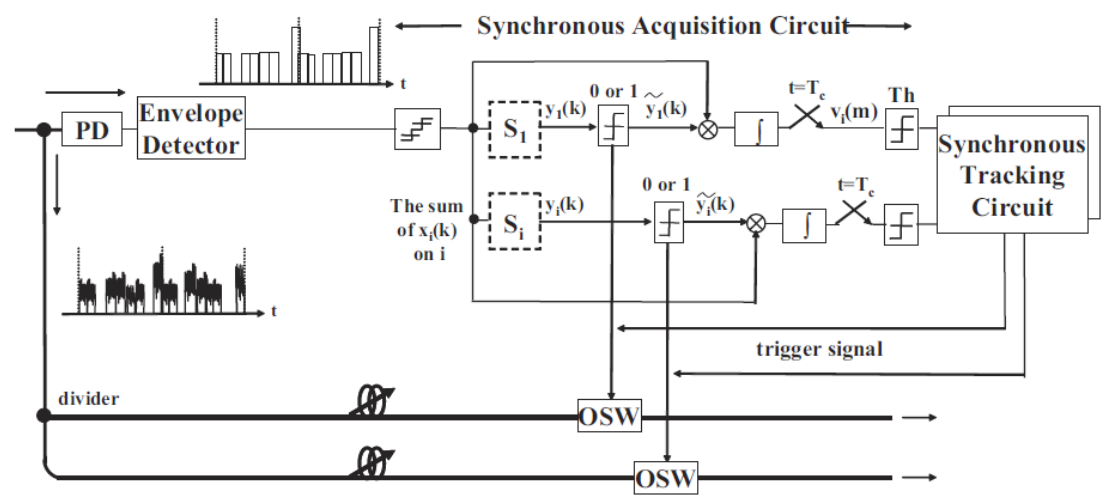

(b)

Fig. 2. Configuration of chaos DOS-CDMA method: (a) RBS and (b) RN

case of not satisfy $T h$, a chip sequence is shifted and synchronous acquisition process is performed again. When the multiple number of wireless services, $M$, increases, the scale of synchronous acquisition circuit becomes still larger by giving the function to which a chip sequence is shifted. Therefore, synchronous acquisition process becomes complicated, and $T_{S A}$ becomes long. A chip sequence is shifted $L$ times at the maximum. $T_{S A}$ is calculated as Eq. (2).

$$
T_{S A}=T_{F} \times \frac{L}{2}
$$

On the other hand, in the case of chaotic sequence, since synchronous acquisition process can be taken only by distinguishing the amplitude value of the received baseband PAM/CDMA signal from any time, shortening of $T_{S A}$ is realized. However, $T_{S A}$ is influenced by the CPU processing speed of the computer. Therefore, $T_{S A}$ is calculated as Eq. (3).

$$
T_{S A}=m \times T_{c}+T_{c o m}
$$

Computer processing time is expressed as $T_{\text {com }}$.

\section{Simulation}

The computer simulation evaluates $T_{S A}$ of the proposed method compared with conventional method which is using sliding correlation and prime code. 


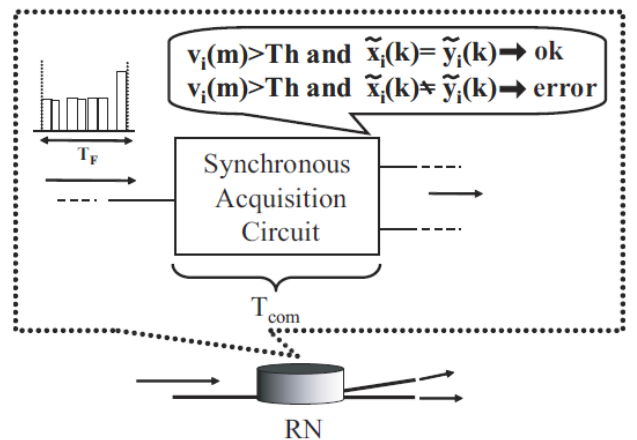

(a)

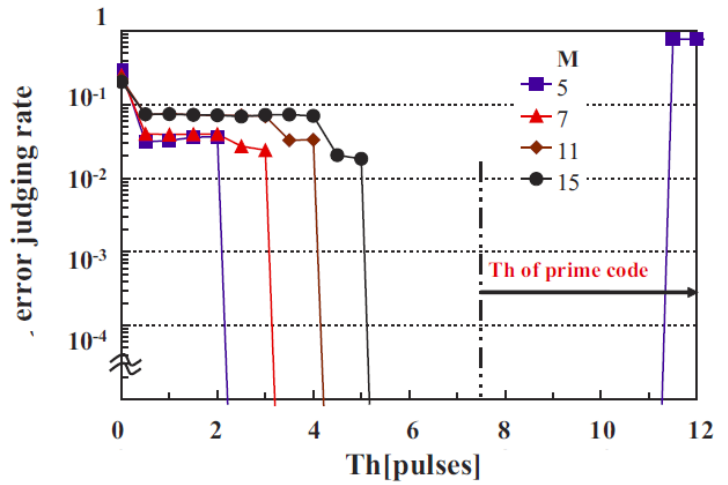

(b)

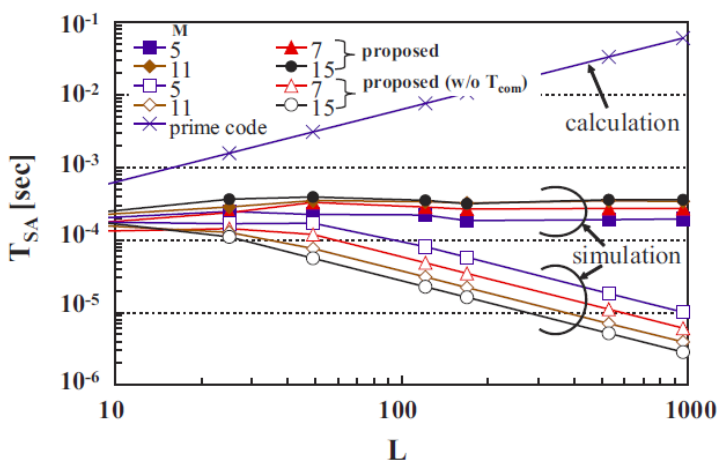

(c)

Fig. 3. Simulations: (a) simulation model, (b) the error judging rate to $T h$ and (c) $T_{S A}$ to $L$

$T_{F}$ is assumed to be $125[\mu \mathrm{sec}]$ since $T_{c}$ fills the band pass sampling theorem in the range more than $1[\mathrm{nsec}]$, when $L$ becomes 1000 or less. In the proposed method, the function based on a tent map was applied to $G_{i}()$, and it was made for the weight to become equivalent with prime code statistically. In this simulation, $T_{\text {com }}$ was estimated by the use of AMD Athlon MP2000+Dual (clock rate of $1.67 \mathrm{GHz}$ ) CPU.

Figure 3 (a) shows the simulation model. It is assumed that $M$ RF signals was received. $T_{c o m}$ is equivalent to the calculation processing time in synchronous acquisition process.

Figure 3 (b) shows the error judging rate to $T h$ for a parameter as $M$. The 
error judging rate needs to set up $T h$ in the range fully near zero. In the case of prime code, $T h$ should set up more than half of weight in consideration of the greatest value of cross correlation. In case of $M=15, T h$ becomes higher than 7.5. In the case of chaotic sequence, $T h$ is enough set as 6.0 when $M$ is 15 or less. Therefore, the proposed method can set up $T h$ low and shorten $T_{S A}$. Also, since the influence of interference by other code sequence becomes large when $M$ increases, $T h$ is highly needed.

Figure 3 (c) shows $T_{S A}$ to $L$ for a parameter as $M$. In case of $L$ of long, $T_{S A}$ of prime code also becomes long. On the other hand, in case of proposed chaotic sequence, $T_{S A}$ is improved as compared with prime code. In case of $L$ of long, $T_{S A}$ becomes almost fixed. This shows that neither $m$ nor $T_{\text {com }}$ is dependent on $L . T_{S A}$ can be reduced by $95 \%$ in case of $L$ is still 121 as compared with the case of prime code, and quick transmission achieved. Since $T_{\text {com }}$ becomes long when $M$ increases, $T_{S A}$ becomes long. Also, when $T_{\text {com }}$ is zero, in case of long $L, T_{S A}$ becomes short since chip speed becomes quick.

\section{Conclusion}

We have proposed a fast synchronous acquisition method which is applied the chaotic synchronization to conventional DOS-CDMA method in the SDRN. Computer simulation shows that synchronous acquisition time couled be reduced by $95 \%$ as compared with the sliding correlation in case of code length of 121 . 